\title{
El análisis del entorno político y legal en el marco de la planificación estratégica en el sector turístico y hotelero
}

\author{
Analysis of the political and legal environment in \\ the context of the strategic planning in tourism and \\ hospitality sector
}

\author{
Maria Teresa Fernández Alles ${ }^{1}$ \\ Universidad de Cádiz (España)
}

Recibido el 25 de febrero de 2010 y aceptado el 9 de marzo de 2010

$\mathrm{N}^{\mathrm{o}}$ de clasificación JEL: J78, L83

DOI: $10.5295 / \mathrm{cdg} .100210 \mathrm{mf}$

\section{Resumen:}

Las empresas turísticas se encuentran inmersas en un entorno cambiante, caracterizado por los avances tecnológicos, al aumento de la competencia global y a las consecuencias derivadas de la crisis económica mundial. Ante estas circunstancias, las empresas turísticas se ven obligadas a diseñar estrategias de marketing $y$ llevar a cabo planes estratégicos. Pues bien, para entender el marketing y desarrollar estrategias eficaces, es fundamental entender el contexto en el que opera la empresa, puesto que en él se generan las oportunidades del mercado y se presentan las amenazas que van a condicionar su futuro. Entre las fuerzas que integran el entorno empresarial se encuentran la economía, la sociedad, la política o la demografía, entre otras. En las últimas décadas, uno de los fenómenos demográficos más relevantes para las empresas turísticas ha sido el aumento de la población discapacitada, junto con una mayor participación de la misma en el ámbito turístico. No obstante, esta participación podría ser aún mayor si los destinos y las empresas turísticas fueran accesibles para las personas discapacitadas. La escasa atención que se les ha prestado, hasta fechas recientes, a las personas discapacitadas y, especialmente, a su derecho al ocio y disfrute de su tiempo libre, han llevado a que los destinos turísticos no sean accesibles para los turistas discapacitados. El presente trabajo está centrado en el estudio de entorno político-legal en el sector turístico, en general, y en el sector hotelero, en particular, en materia de accesibilidad.

Palabras clave:

Entorno Político-Legal, Discapacidad, Accesibilidad, Normativa.

Abstract:

\footnotetext{
${ }^{1}$ Profesora Doctora de la Universidad de Cádiz, Facultad de Ciencias Económicas y Empresariales, Departamento de Marketing y Comunicación, Glorieta Carlos Cano s/n. 11002 Cádiz. Email: teresa.alles@uca.es
} 
Tourism companies are caught in a changing environment, characterized by technological advances, increased global competition and the consequences of the global economic crisis. Under these circumstances, tourism companies are forced to devise marketing strategies and implement strategic plans. Well, to understand the marketing and developing effective strategies is to understand the context in which the company operates, since it will generate market opportunities and threats presented that will condition their future. Among the forces that comprise the business environment are the economy, society, politics, demographics, among others. One of the most significant demographic phenomena for tourism enterprises in the last decade has been the increase in the disabled population, combined with greater participation in the tourism sector itself. However, this participation could be even greater if both destinations and companies comprising it were accessible to the disabled. The scant attention paid to them until recently, disabled people, and especially their right to leisure and enjoy your free time, have led to tourist destinations not accessible to disabled tourists. The present work is focused on the study of political and legal environment in the tourism sector in general and in the hotel sector, particularly regarding accessibility.

\section{Keywords:}

Political-Legal Environment, Disability, Accessibility, Standards. 


\section{INTRODUCCIÓN}

Desde el punto de vista del marketing, el objetivo fundamental de las empresas es crear relaciones duraderas con los clientes, como vía para lograr el éxito en el mercado. Para ello, las empresas llevan a cabo un proceso mediante el cual "crean valor para los clientes y establecen relaciones sólidas con ellos obteniendo a cambio el valor de los clientes" (Kotler y Armstrong, 2008, 5).

Este proceso comprende una serie de etapas, siendo el punto de partida el conocimiento del mercado en el que actúa o va a actuar la empresa, el descubrimiento de las necesidades y deseos de los consumidores que lo integran y el análisis del entorno cambiante y complejo en el que opera la empresa.

Este entorno está formado por una serie de agentes (intermediarios, clientes, competidores, etc.) y fuerzas (demográficas, económicas, políticas, culturales, tecnológicas, etc.) que influyen directamente en su capacidad para desarrollar y mantener con éxito relaciones a largo plazo con sus clientes. Desde el punto de vista del marketing, es fundamental la observación de este entorno como vía para poder adaptarse a los cambios que acontecen en él, y como medio para garantizar el éxito de la empresa. Esto es debido a que en el entorno es donde se generan las oportunidades y las amenazas que influyen en la actuación empresarial. Por ello, el análisis del entorno es una fase clave en el proceso de planificación estratégica de marketing.

En el sector turístico, el estudio del entorno demográfico, y de manera específica, el análisis poblacional, constituye un elemento clave del análisis del entorno puesto que los mercados turísticos están integrados por personas, y las personas, es decir, los turistas, son la pieza clave del sector turístico.

Desde el punto de vista turístico, uno de los fenómenos demográficos más relevantes de las últimas décadas, junto con el aumento del número de habitantes y el envejecimiento poblacional, es el incremento de la población discapacitada, hecho que ha ido acompañado de una mayor participación de las personas discapacitadas en el ámbito turístico. El aumento de las ayudas técnicas y económicas ha propiciado el aumento de viajes realizados por personas con discapacidad, convirtiendo al "turista discapacitado" en una oportunidad de negocio para los destinos y las empresas turísticas.

Actualmente, el número de personas discapacitadas asciende a 600 millones en todo el mundo y 50 millones en Europa, de las cuales casi 40 millones viven en algún país de la Unión Europea (O.M.S., 2009). Según la Encuesta de Discapacidad, Autonomía Personal y Situaciones de Dependencia (EDAD) (I.N.E., 2009), el número de personas discapacitadas, en España, asciende a un total de 3.847.900, representando el 8,5\% de la población (INE, 2009).

No obstante, si hacemos referencia al número de personas que se beneficiarían de la supresión de barreras el volumen poblacional sería mucho mayor. Así, según la Encuesta sobre Discapacidades, Deficiencias y Estado de la Salud (EDDES-99), dentro del segmento de los discapacitados habría que incluir no sólo a los discapacitados permanentes (motrices, sensoriales y mentales), a los que hacen referencia las cifras anteriores, sino, además, a las personas mayores de 60 años sin discapacidades y a los discapacitados temporales, entre los que se incluyen a las mujeres embarazadas y a las personas enyesadas, grupos que representan el $40 \%$ de la población. 
Teniendo en cuenta que no todas las personas con discapacidad viajan, ya sea porque padecen discapacidades muy severas, precisando altos servicios especializados, o por la falta de recursos económicos suficientes para hacer turismo, se puede estimar el mercado potencial para la industria turística accesible en Europa en el 72\% de la población con discapacidad, unos 36 millones de personas (CERMI, 2005).

Según el documento "Accesibilidad a Turistas con Discapacidades", la demanda potencial de viajes de los europeos con discapacidades se puede estimar en (DG XIII de la Comisión Europea):

- 8 millones que viajarían al extranjero.

- 15 millones los que viajarían dentro del país.

- 22 millones de excursionistas locales de un día.

Lo que supondrían un potencial de 35 millones de viajeros que pernoctan y 630 millones de noches de hotel cada año. A lo que habría que añadir el hecho de que, según publica la Comisión Europea (1991) en el documento "Por una Europa Accesible a turistas con Discapacidades", se calcula que cada viaje realizado por una persona discapacitada atrae a 0,5

acompañantes. No obstante, debemos tener en cuenta que no todas las personas discapacitadas pueden viajar, ya sea por no disponer de suficientes recursos económicos para hacer turismo y/o padecer discapacidades que les impiden viajar.

Pues bien, a pesar del aumento experimentado, en los últimos años, en el volumen de turistas discapacitados en el mercado turístico, hoy en día, son muchas las personas discapacitadas que no viajan, no por falta de tiempo o deseos de viajar, sino por temor a no encontrar destinos turísticos accesibles ${ }^{1}$, es decir, sin barreras, que les garanticen la comodidad, seguridad y autonomía que demandan en sus viajes.

Las razones que han llevado a esta situación de inaccesibilidad en el turismo parecen venir justificadas, en parte, por la escasa atención que se les ha prestado, hasta fechas recientes, a las personas discapacitadas y, especialmente, a su derecho al ocio y disfrute de su tiempo libre.

Por ello, el presente trabajo está centrado en el estudio del entorno político en materia de accesibilidad, teniendo en cuenta que el entorno político es el que está integrado por las "leyes, dependencias del gobierno y grupos de presión que influyen en diversas organizaciones e individuos de una sociedad determinada y los limitan" (Kotler y Armstrong, 2008, 83). Concretamente, analizaremos la normas que regulan la accesibilidad en el sector turístico, en general, y en el sector hotelero, en particular.

Los objetivos perseguidos con este trabajo son múltiples:

- En primer lugar, proteger a las empresas turísticas y hoteleras desde un punto de vista legal y competitivo, a través del cumplimiento de la normativa accesible. La eliminación de barreras en los establecimientos turísticos y hoteleros no sólo contribuirá al cumplimiento de la normativa legal, sino que constituirá un factor de diferenciación frente a los establecimientos no accesibles, pudiendo ser considerado un factor de competitividad en el mercado turístico a tener en cuenta en el diseño de las estrategias de marketing.

\footnotetext{
${ }^{1}$ La accesibilidad hace referencia al "conjunto de características de que debe disponer un entorno, producto o servicio para ser utilizable en condiciones de confort, seguridad e igualdad por todas las personas y, en particular, por aquellas que tienen alguna discapacidad" (IMSERSO, Libro de la Accesibilidad, 2003, 20).
} 
- En segundo lugar, contribuir al logro de destinos y alojamientos turísticos accesibles, evitando, así, que los turistas discapacitados sean objeto de un trato desigual. La eliminación de barreras en las instalaciones turísticas y hoteleras evitará que las personas discapacitadas sean discriminadas cuando viajan;

- Por último, proteger a la sociedad en su conjunto de comportamientos empresariales no responsables que limita el acceso de las personas discapacitadas y de sus familiares al sector turístico, mermando la calidad de vida de los mismos.

\section{EL ENTORNO POLÍTICO-LEGAL EN MATERIA DE ACCESIBILIDAD}

En las últimas décadas han surgido iniciativas, tanto públicas como privadas, en favor del derecho de estas personas a la igualdad de trato en el ocio y el turismo, lo que ha provocado una mayor concienciación en diversos ámbitos empresariales, políticos y sociales. Concretamente, en las dos últimas décadas se han aprobado múltiples disposiciones y reglamentaciones destinadas a lograr un entorno accesible para las personas discapacitadas, evitando así que las mismas sean objeto de un trato desigual.

Desde hace años, las Naciones Unidas vienen luchando por mejorar la calidad de vida de las personas con discapacidad, lo que ha quedado patente en sus principios fundamentales, basados en los derechos humanos, las libertades fundamentales y la igualdad de todas las personas.

El artículo $1^{\circ}$ de la Declaración de los Derechos Humanos, sobre el derecho a la igualdad para todos los humanos, la protección contra la discriminación y la participación en la vida de la comunidad, expresa que "todos los seres humanos son libres e iguales en dignidad y derechos"... “A fin de alcanzar esta meta, todas las comunidades deben celebrar la diversidad en el seno de sí mismas, y deben asegurar que las personas con discapacidad puedan disfrutar de las distintas clases de derechos humanos: civiles, políticos, sociales, económicos y culturales, reconocidos por las distintas Convenciones internacionales, el Tratado de la Unión Europea y en las constituciones nacionales".

A raíz de la celebración en Suiza, en 1963, del Congreso Internacional para la Supresión de Barreras Arquitectónicas, se elaboran una serie de informes, comunicaciones y declaraciones sobre la materia, destacando la Resolución 3447, adoptada por la Asamblea General de las Naciones Unidas el 9 de diciembre de 1975, denominada "Declaración de los Derechos de las Personas Discapacitadas", referida a los derechos civiles y políticos de las personas discapacitadas.

En 1976, los Estados Miembros de las Naciones Unidas, con el objetivo de promover la completa participación e igualdad, deciden proclamar el año 1981 como el "Año Internacional de las Personas Discapacitadas".

En 1982, la Asamblea General de las Naciones Unidas aprueba el "Programa Mundial de Acción concerniente a las Personas Discapacitadas", en la lucha por los derechos de las personas con minusvalías a disfrutar de las mismas oportunidades que las personas que no las padecen.

Pero, no es hasta finales de los años 80 y principios de los 90 , cuando se produce un verdadero cambio en las políticas internacionales en materia de accesibilidad debido, fundamentalmente, a la introducción del concepto "Diseño para Todos", por una parte, y a la 
presión realizada por personas e instituciones para lograr la igualdad de trato de las personas con y sin discapacidad, por otra.

En Diciembre de 1993, por Resolución de la Asamblea General de las Naciones Unidas, se aprueban las "Normas Uniformes sobre la Igualdad de Oportunidades para las personas con Discapacidad" (ONU, 1996). Estas Normas, aunque no son de obligado cumplimiento, desde el punto de vista jurídico, suponen un compromiso en la adopción de medidas, así como un compromiso en el desarrollo de políticas por la igualdad de oportunidades de las personas discapacitadas. Destacamos los artículos 5 y 14 que establecen lo siguiente:

- Artículo 5": "Para las personas con discapacidades de cualquier índole, los Estados debe establecer programas de acción para que el entorno sea accesible, y adoptar medidas para garantizar el acceso a la información y la comunicación".

- Artículo 14: "Los Estados deben emprender y prever políticas adecuadas para las personas con discapacidad en el plano nacional y deben estimular y apoyar medidas en los planos regional y local".

Finalmente, debemos destacar la Convención sobre los derechos de las personas con discapacidad, adoptada por Naciones Unidas el 13 de diciembre de 2006 (ratificada por España el 23 de noviembre de 2007 y publicada en el BOE el 21 de abril de 2008). Entre sus objetivos destacan el promover, proteger y asegurar el goce pleno y en condiciones de igualdad de todos los derechos humanos y libertades fundamentales para todas las personas con discapacidad.

Por todo lo expuesto anteriormente, consideramos fundamental analizar el entorno político-legal en el ámbito turístico, desde la óptica de la accesibilidad, estudiando las normas que deben guiar la actuación de las empresas turísticas en materia de accesibilidad y no discriminación de los turistas por razón de discapacidad.

\subsection{La accesibilidad en el ambito de la unión europea}

El primer programa europeo, dirigido específicamente a las personas con discapacidad, que sirvió de base para la posterior creación de una política social en materia de discapacidad, fue el Programa de acción comunitaria para la readaptación profesional de los minusválidos, aprobado en 1974. Pero, es en los años 80, cuando las instituciones europeas comienzan a dedicar una atención creciente a la discapacidad, lo que queda reflejado en la diversidad de documentos y directrices aprobadas, entre las que cabe destacar las siguientes:

- Resolución del Parlamento Europeo sobre la integración económica, social y profesional de los minusválidos en la Comunidad, de 11 de mayo de 1981.

- Comunicación de la Comisión sobre las líneas directrices de una acción comunitaria para la inserción social de los minusválidos, de 4 de noviembre de 1981.

- Resolución del Consejo y de los Representantes de los Gobiernos de los Estados miembros reunidos en la sede del Consejo sobre la integración social de los minusválidos, de 21 de diciembre de 1981, entre otras.

En el año 1988 se puso en marcha el programa HELIOS, Segundo programa de acción de la Comunidad a favor de los minusválidos, de aplicación durante el cuatrienio 1988-1991, siendo uno de sus objetivos fundamentales la igualdad de oportunidades y la 
vida independiente de las personas con discapacidad. Años más tarde, en 1993, se aprueba el programa HELIOS II, Tercer programa de acción comunitaria para las personas con minusvalías, para el cuatrienio 1993-1996, destacando entre algunos de sus objetivos la integración económica y social, así como la mejora de la autonomía.

Sobre la experiencia adquirida con los programas HELIOS I y II, la Comisión Europea adoptó, el 30 de julio de 1996, la Comunicación sobre Igualdad de Oportunidades para las Personas con Minusvalías, en la que se define una nueva estrategia comunitaria en materia de discapacidad. La Comunicación, basada en el principio de igualdad de oportunidades, no discriminación, derechos, normalización, inclusión y plena participación, respalda los planteamientos recogidos en la Normas Uniformes sobre Igualdad de Oportunidades para las Personas con Discapacidad (1993), elaboradas por las Naciones Unidas, afirmando la necesidad de un nuevo enfoque centrado en la identificación y supresión de las barreras, ya que se oponen a la igualdad de oportunidades y a la plena participación de las personas con discapacidad.

En ese mismo año, se redacta el Concepto Europeo de Accesibilidad, documento que ha supuesto un verdadero avance en materia de accesibilidad, y la Unidad de Turismo, de la Dirección General XXIII, publica el documento "Por una Europa accesible a turistas con discapacidad", con el objeto de formar a los profesionales del turismo de Europa sobre cómo hacer accesibles sus establecimientos y cómo tratar a los clientes con discapacidad.

En el año 1997, el Consejo de la Unión Europa aprueba el Tratado de Ámsterdam, incluyendo en su articulado aspectos relacionados con la discriminación, destacando los artículos 6 y 13, según los cuales los países de la Unión Europea deberán adoptar planes de acción para la integración social de las personas discapacitadas.

Siguiendo las directrices del artículo 13, la Comisión Europea redacta, en el año 2000, la Comunicación Hacia una Europa Sin Barreras para las Personas con Discapacidad, con el objetivo de alcanzar la accesibilidad de los discapacitados a cualquier entorno, planteándose la revisión de algunas políticas fundamentales de la Unión Europea, en la búsqueda de unas mejores condiciones de acceso de las personas con discapacidad.

En el seno del Congreso Europeo sobre Discapacidad, celebrado en Madrid en marzo de 2002, se firmó la Declaración de Madrid “No Discriminación más Acción Positiva es Igual a Inclusión Social”, en la que se recogían una serie de propuestas de acción que se concretaban en ocho puntos relativos a la no discriminación en el acceso a los servicios ordinarios de salud, educativos, profesionales y sociales, así como a todas las oportunidades disponibles para las personas no discapacitadas, incluyendo a todos los agentes involucrados en la consecución de este objetivo. Con esta Declaración se pretendía proporcionar un marco conceptual de acción, de cara a la celebración, en el año 2003, del "Año Europeo de las Personas con Discapacidad” en el ámbito de la Unión Europea, nacional, regional y local, con un objetivo claro, alcanzar la plena igualdad de oportunidades, conscientes, por un lado, de que las bases fundamentales de nuestra sociedad son el respeto de la diversidad humana y la equiparación de los derechos de todas las personas, y, por otra parte, que las barreras en el entorno constituyen obstáculos más graves que las limitaciones funcionales de las personas. Ambos conceptos, igualdad de oportunidades y diseño para todos, son las dos grandes ideas en las que se basa la lucha por la accesibilidad en los últimos años.

En el mismo año 2003, la Dirección General de Empresas de la Comisión Europea publica un documento conteniendo una serie de "Criterios armonizados por una buena ac- 
cesibilidad de los lugares e infraestructuras turísticas para las personas con discapacidad". También hay que destacar, de ese mismo año, el Dictamen del Comité Económico y Social Europeo "Por un turismo accesible a todas las personas y socialmente sostenible", aprobado el 29 de octubre de 2003.

En el año 2006, el Consejo de Europa crea el Comité de Expertos en Diseño Universal, del que forman parte representantes de los Estados Miembros del Consejo. Se trata de un grupo de trabajo denominado "Universal Design (Accesibility) for People with Disabilities".

Ese mismo año se aprueba la Recomendación Rec(2006)5 del Comité de Ministros de los Estados Miembros sobre el "Plan de Acción del Consejo de Europa para la promoción de derechos y la plena participación de las personas con discapacidad en la sociedad: mejorar la calidad de vida de las personas con discapacidad en Europa 2006-2015" (adoptada por el Comité de Ministros el 5 de abril de 2006,durante la $961^{a}$ reunión de Delegados de Ministros. Esta Recomendación, en atención a la Resolución (96) 35 de 2 de octubre de 1996, tiene como objetivo proseguir las actividades llevadas a cabo y desarrolladas en virtud de aquella Resolución, "encaminadas a la integración de las personas con discapacidad en la sociedad, con el fin de definir y contribuir a aplicar a nivel europeo un modelo de política coherente para las personas con discapacidad, basado en los principios de plena ciudadanía y de vida autónoma, lo que implica la eliminación de obstáculos a la integración, sean de la naturaleza que sean, psicológica, educativa, familiar, cultural, social, profesional, financiera o arquitectónica" (Consejo de Europa, 2006).

Los principios fundamentales que regula el presente Plan de Acción son los siguientes: no discriminación; igualdad de oportunidades; plena participación en la sociedad de todas las personas con discapacidad; respeto a la diferencia y reconocimiento de la discapacidad como componente de la diversidad humana; dignidad y autonomía del individuo, incluida la libertad de hacer sus propias elecciones; igualdad entre hombres y mujeres; y participación de las personas con discapacidad en todas las decisiones sobre su vida, tanto a nivel individual como a nivel de la sociedad en su conjunto, a través de sus organizaciones representativas.

Finalmente, debemos mencionar la Resolución del Consejo de la Unión Europea y los Representantes de los Gobiernos de los Estados miembros, reunidos en el Consejo el 17 de marzo de 2008, relativa a la situación de las personas con discapacidad en la Unión Europea. Esta Resolución tiene como objetivo fundamental recordar la Convención de las Naciones Unidas sobre los derechos de las personas con discapacidad y el Plan de acción europeo en materia de discapacidad 2004-2010, reconociéndose la situación de desventaja que todavía padecen muchas personas con discapacidad.

\subsection{La accesibilidad en España}

Las leyes de accesibilidad en España encuentran su fundamento en los artículos 9.2, 14 y 49 de la Constitución Española. El primero de ellos, el artículo 9.2, establece que los poderes públicos promoverán las condiciones para que la igualdad del individuo y de los grupos en que se integra sea real y efectiva; removerán los obstáculos que impidan o dificulten su plenitud y facilitarán la participación de todos los ciudadanos en la vida política, 
económica, cultural y social. La finalidad es alcanzar la igualdad real, no sólo la igualdad formal, que establece el artículo 14, en el que se reconoce la igualdad de todos los españoles ante la ley, sin que pueda prevalecer discriminación alguna.

El artículo 49 determina que "los poderes públicos realizarán una política de previsión, tratamiento, rehabilitación e integración de las personas con deficiencias físicas, sensoriales y psíquicas, a los que prestarán atención especializada que requieran y los ampararán especialmente para el disfrute de los derechos que en su Título Primero otorga a todos los ciudadanos".

Con el fin de alcanzar los objetivos marcados en estos artículos, se promulgó la Ley 13/1982, de 7 de abril, de Integración Social de los Minusválidos (LISMI) (B.O.E., nº 103, de 30 de Abril), la cual significó un avance importante en la mejora de las condiciones de vida de las personas con discapacidad en España. En ella, destacamos el artículo 54.1 en el que se establece que "la construcción, ampliación y reforma de los edificios de propiedad pública o privada, destinados a un uso que implique la concurrencia de público, así como la planificación y urbanización de las vías públicas, parques y jardines de iguales características, se efectuará de forma tal que resulten accesibles y utilizables a los minusválidos”.

A raíz de esta ley se aprobaron los siguientes Reales Decretos en materia de accesibilidad:

- Real Decreto 556/1989, de 19 de Mayo, por el que se arbitran medidas mínimas de accesibilidad en los edificios. Destacamos, en el mismo, los siguientes artículos:

- Art. $1^{\circ}$. Dispone que en los nuevos edificios de concurrencia pública tendrán que ser practicables por personas con problemas de movilidad, al menos, los siguientes itinerarios: la comunicación entre el interior y el exterior del edificio, y entre éste y las áreas de uso público; además, un aseo estará adaptado para su uso por personas con movilidad reducida.

- Art. $2^{\circ}$. Recoge una serie de condiciones mínimas que deberá cumplir un itinerario para ser considerado practicable por las personas con problemas de movilidad.

- Real Decreto 19/1989, de 5 de Mayo, sobre medidas mínimas de accesibilidad en los edificios, obras públicas y urbanismo.

- Real Decreto 775/2002, de 26 de Julio, por el que se crea el Comité Español de Coordinación para el Año Europeo de las Personas con Discapacidad.

La Ley 13/1982, de 7 de abril, de Integración Social de los Minusválidos (LISMI) fue derogada con la aprobación de la Ley 51/2003, de 2 de diciembre, de Igualdad de Oportunidades, No Discriminación y Accesibilidad Universal de las Personas con Discapacidad ${ }^{2}$, teniendo como objetivos básicos el establecimiento de medidas que garanticen y hagan efectivo el derecho a la igualdad de las personas con discapacidad fijados en los artículos 9.2, 10, 14 y 49 de la Constitución, fijando, asimismo, las pautas de actuación en materia de discapacidad. Esta ley establece como principios fundamentales, que han de guiar las políticas y decisiones públicas en relación con la discapacidad, los siguientes: la vida inde-

\footnotetext{
${ }^{2}$ La accesibilidad hace referencia al "conjunto de características de que debe disponer un entorno, producto o servicio para ser utilizable en condiciones de confort, seguridad e igualdad por todas las personas y, en particular, por aquellas que tienen alguna discapacidad" (IMSERSO, Libro de la Accesibilidad, 2003, 20).
} 
pendiente, la accesibilidad universal, el diseño para todos, y el diálogo civil y la transversalidad de las políticas en materia de discapacidad, entre otros.

Por otra parte, recoge el derecho a la igualdad de oportunidades de las personas con discapacidad, así como la ausencia de discriminaciones, exigiéndose la accesibilidad y la realización de ajustes razonables y medidas de acción positiva destinadas a compensar las especiales dificultades que tienen las personas con discapacidad para su plena participación en la vida social. Además, establece la obligación gradual y progresiva de todos los entornos, productos y servicios, de ser abiertos, accesibles y practicables para todas las personas. Para ello, dispone plazos y calendarios para la realización de las adaptaciones necesarias en cumplimiento de las obligaciones establecidas en materia de accesibilidad y no discriminación.

La Ley 51/2003, de 2 diciembre, de Igualdad de Oportunidades, no Discriminación y Accesibilidad Universal de las Personas con Discapacidad, en sus disposiciones finales, contiene mandatos explícitos de desarrollo y aplicación de las medidas determinadas por la ley. Para dar respuesta a estos mandatos, se han elaborado y publicado los siguientes reales decretos:

- Real Decreto 1414/2006, de 1 de diciembre, por el que se determina la Consideración de Persona con Discapacidad a los efectos de la Ley 51/2003 de Igualdad de Oportunidades, no Discriminación y Accesibilidad Universal de las Personas con Discapacidad (BOE de 16 de diciembre de 2006).

- Real Decreto 1417/2006, de 1 de diciembre, por el que se establece el Sistema Arbitral para la Resolución de Quejas y Reclamaciones en Materia de Igualdad de Oportunidades, no Discriminación y Accesibilidad por Razón de Discapacidad. (BOE de 13 de diciembre de 2006).

- Real Decreto 366/2007, de 16 de marzo, por el que se establecen las Condiciones de Accesibilidad y no Discriminación de las Personas con Discapacidad en sus Relaciones con la Administración General del Estado. (BOE de 24 de marzo de 2007).

- Real Decreto 1544/2007, de 23 de noviembre, por el que se regulan las condiciones básicas de accesibilidad y no discriminación para el acceso y utilización de los modos de transporte para personas con discapacidad. (BOE de 4 de diciembre de 2007)

- Ley 49/2007, de 26 de diciembre, por la que se establece el régimen de infracciones y sanciones en materia de igualdad de oportunidades, no discriminación y accesibilidad universal de las personas con discapacidad. (BOE de 27 de diciembre de 2007)

El artículo 149.1.1 a de la Constitución establece que "el Estado tiene competencia exclusiva sobre la regulación de las condiciones básicas que garanticen la igualdad de todos los españoles en el ejercicio de los derechos y en el cumplimiento de los deberes constitucionales". Como establece el artículo 10 de la ley 51/2003, "el Gobierno, sin perjuicio de las competencias atribuidas a la comunidades autónomas y a las corporaciones locales, regulará unas condiciones básicas de accesibilidad". No obstante, según las materias que les han sido transferidas en virtud del artículo 148 de la Constitución Española, y lo establecido en sus estatutos de autonomía, las Comunidades Autónomas cuentan con su propia normativa en materia de accesibilidad y supresión de barreras.

Así, desde mediados de los años 80, la práctica totalidad de las Comunidades Autónomas han venido publicando su propia normativa en materia de supresión de barreras arquitectónicas, urbanísticas y de transportes, estableciendo parámetros técnicos específicos que 
varían de una a otra Comunidad. En el anexo se incluye toda la normativa y los reglamentos vigentes en la actualidad en las Comunidades Autónomas españolas.

\section{CONCLUSIONES}

El análisis estratégico es una fase previa en el proceso de planificación, que permite descubrir las amenazas y oportunidades que el entorno presenta a la organización, así como las debilidades y fortalezas que muestra la empresa en relación a sus competidores. Concretamente, el análisis del entorno empresarial es considerado una pieza clave en el proceso de planificación de marketing. Este análisis debe estar centrado en las múltiples fuerzas, demográficas, económicas, sociales, políticas, tecnológicas o culturales, en el que se desarrolla la actividad empresarial.

De especial relevancia es la importancia estratégica de las personas discapacitadas para el sector turístico, en general, y el sector hotelero, en particular, puesta de manifiesto en el estudio del entorno demográfico, lo que queda reflejado, en primer lugar, el aumento del número de personas discapacitadas en el conjunto poblacional, y en segundo lugar, en su creciente participación en el sector turístico, propiciada por la existencia de un mayor número de ayudas técnicas y económicas.

No obstante, en la actualidad, son muchas las personas discapacitadas que no viajan debido a la existencia de múltiples las barreras en los destinos turísticos, a pesar de que la accesibilidad, o lo que es lo mismo, la ausencia de barreras, es considerada no sólo una necesidad básica de los personas discapacitadas, sino, además, un factor determinante de su nivel de satisfacción como consumidores turísticos.

De ahí que consideremos de vital importancia para las empresas turísticas la eliminación de barreras, como condición indispensable para lograr la incorporación de las personas discapacitadas en el ámbito turístico en condiciones de igualdad y sin discriminación alguna respecto al resto de la población.

Para ello, es preciso que los propietarios y dirigentes de las empresas turísticas realicen un estudio del entorno político en materia de accesibilidad, como vía para conocer las múltiples disposiciones y reglamentaciones aprobadas en el ámbito de la Unión Europea, en general, y en España y la Comunidad Autónoma en la que están ubicadas, en particular.

Consideramos que el conocimiento de la normativa aplicable en materia de accesibilidad, por parte de los agentes turísticos, tanto públicos como privados, es el punto de partida para lograr que los destinos turísticos accesibles y que en ellos se pueda garantizar la comodidad, seguridad y autonomía que demanda los turistas discapacitados. Sólo a través del conocimiento y el cumplimiento de las normas que regulan la accesibilidad en el turismo, las personas discapacitadas podrán ejercitar su derecho de acceso al ocio y al turismo, en las mismas condiciones al resto de la población y sin discriminaciones.

Por otra parte, las empresas turísticas, con la incorporación de la accesibilidad en sus instalaciones, a través de la eliminación de las barreras, podrán mejorar su imagen, no sólo desde el punto de vista de la calidad, sino, también, desde el punto de vista social, al no limitar su oferta a ningún segmento de mercado por razón de discapacidad. 


\section{ANEXO}

\section{ANDALUCÍA}

- Ley 1/1999, de 31 de marzo, de Atención a las Personas con Discapacidad en Andalucía. (B.O.J.A. de 17-4-99).

- Decreto 293/2009, de 7 de julio, por el que se aprueba el reglamento que regula las normas para la accesibilidad en las infraestructuras, el urbanismo, la edificación y el transporte en Andalucía. (B.O.J.A. de 21-7-09).

\section{ARAGÓN}

- $\quad$ Ley 3/1997, de 7 de abril, de Promoción de la Accesibilidad y Supresión de Barreras Arquitectónicas, Urbanísticas, de Transportes y de la Comunicación. (B.O.A. de 18-4-97).

- Decreto 19/1999, de 9 de febrero, del Gobierno de Aragón, por el que se regula la Promoción de la Accesibilidad y Supresión de Barreras Arquitectónicas, Urbanísticas, de Transportes y de la Comunicación. (B.O.A. de 15-3-99). Modificado por el Decreto 108/2000, de 29 de mayo (B.O.A. de 7-6-00) y la Orden de 15-6-00 (B.O.A. de 5-7-00).

\section{ASTURIAS}

- $\quad$ Ley 5/1995, de 6 de abril, de promoción de la accesibilidad y supresión de barreras. (B.O.P.A. de 19-4-95).

- Decreto 37/2003. de 22 de mayo, por el que se aprueba el Reglamento de la Ley 5/1995, de 6 de abril, de promoción de la accesibilidad y supresión de barreras, en los ámbitos arquitectónico y urbanístico. (B.O.P.A. de 11-6-03).

\section{BALEARES}

- $\quad$ Ley 3/1993, de 4 de mayo, para la mejora de la accesibilidad y de la supresión de las barreras arquitectónicas. (B.O.C.A.I.B. de 20-5-93).

- Decreto 20/2003, de 28 de febrero, por el que se aprueba el Reglamento de Supresión de Barreras Arquitectónicas. (B.O.I.B. de 18-3-03).

\section{CANARIAS}

- Ley 8/1995, de 6 de abril, de Accesibilidad y Supresión de Barreras Físicas y de la Comunicación. (B.O.C.A.C. de 24-4-95).

- Decreto 227/1997, de 18 de septiembre, por el que se aprueba el Reglamento de la Ley 8/1995, de 6 de abril, de accesibilidad y supresión de barreras físicas y de la comunicación. (B.O.C.A.C. de 21-11-97). Modificado por el Decreto 148/2001, de 9 de julio. (B.O.C.A.C. de 18-7-01).

\section{CANTABRIA}

- Decreto 61/1990, de 6 de julio, sobre evitación y supresión de barreras arquitectónicas y urbanísticas en Cantabria. (B.O.C. de 29-11-90).

- Ley de Cantabria 3/1996, de 24 de septiembre, sobre Accesibilidad y Supresión de Barreras Arquitectónicas, Urbanísticas y de la Comunicación. (B.O.C. de 2-10-96).

\section{CASTILLA - LA MANCHA}

- Ley 1/1994, de 24 de mayo, de Accesibilidad y Eliminación de Barreras en Castilla-La Mancha. (D.O.C.M. de 24-6-94).

\section{CASTILLA Y LEÓN}

- $\quad$ Ley 3/1998, de 24 de junio, de Accesibilidad y Supresión de Barreras. (B.O.C.y L. de 1-798). Modificada por la Ley 11/2000, de 28 de diciembre. (B.O.C.y L. de 30-12-00).

- Decreto 217/2001, de 30 de agosto, por el que se aprueba el Reglamento de Accesibilidad y Supresión de Barreras. (B.O.C.y L. de 4-9-01).

\section{CATALUÑA}

- Ley 20/1991, de 25 de noviembre, de promoción de la accesibilidad y de supresión de barreras arquitectónicas. (D.O.G.C. de 4-12-91). 
- Decreto 135/1995, de 24 de marzo, de desarrollo de la Ley 20/1991, de 25 de noviembre, de promoción de la accesibilidad y de supresión de barreras arquitectónicas, y de aprobación del Código de accesibilidad. (D.O.G.C. de 28-4-95). Modificado por el Decreto 97/2002, de 5 de marzo. (D.O.G.C. de 25-3-02).

\section{EXTREMADURA}

- Ley 8/1997, de 18 de junio, de Promoción de la Accesibilidad en Extremadura. (D.O.E. de 3-7-97). Modificada por la Ley 6/2002, de 27 de junio. (D.O.E. de 23-7-02).

- Decreto 8/2003, de 28 de enero, por el que se aprueba el Reglamento de la Ley de Promoción de la Accesibilidad en Extremadura. (D.O.E. de 20-3-03).

\section{GALICIA}

- $\quad$ Ley 8/1997, de 20 de agosto, de accesibilidad y supresión de barreras en la Comunidad Autónoma de Galicia. (D.O.G. de 29-8-97).

- Decreto 35/2000, de 28 de enero, por el que se aprueba el Reglamento de desarrollo y ejecución de la Ley de accesibilidad y supresión de barreras en la Comunidad Autónoma de Galicia. (D.O.G. de 29-2-00).

\section{LA RIOJA}

- Ley 5/1994, de 19 de julio, de supresión de barreras arquitectónicas y promoción de la accesibilidad. (B.O.L.R. de 23-7-94).

- Decreto 19/2000, de 28 de abril, por el que se aprueba el Reglamento de Accesibilidad en relación con las Barreras Urbanísticas y Arquitectónicas, en desarrollo de la Ley 5/1994, de 19 de julio. (B.O.L.R. de 3-6-00).

\section{MADRID}

- Ley 8/1993, de 22 junio, de Promoción de la Accesibilidad y Supresión de Barreras Arquitectónicas. (B.O.C.M. de 29-6-93).

- Decreto 138/1998, de 23 de julio, por el que se modifican determinadas especificaciones técnicas de la Ley 8/1993, de 22 de junio, de Promoción de la Accesibilidad y Supresión de Barreras Arquitectónicas. (B.O.C.M. de 30-7-98).

- Decreto 13/2007, de 15 de marzo, del Consejo de Gobierno, por el que se aprueba el Reglamento Técnico de Desarrollo en Materia de Promoción de la Accesibilidad y Supresión de Barreras Arquitectónicas. (B.O.C.M. de 24-4-07).

\section{MURCIA}

- Orden de 15 de octubre de 1991, de la Consejería de Política Territorial, Obras Públicas y Medio Ambiente, sobre accesibilidad en espacios públicos y edificación. (B.O.R.M. de 1111-91).

\section{NAVARRA}

- $\quad$ Ley Foral 4/1988, de 11 de julio, sobre barreras físicas y sensoriales. (B.O.N. DE 15-7-88).

- Decreto Foral 154/1989, de 29 de junio, por el que se aprueba el Reglamento para el desarrollo y aplicación de la Ley Foral 4/1988, de 11 de julio, sobre barreras físicas y sensoriales. (B.O.N. de 21-7-89).

\section{PAÍS VASCO}

- $\quad$ Ley 20/1997, de 4 de diciembre, para la Promoción de la Accesibilidad. (B.O.P.V. de 24-1297).

- Decreto 68/2000, de 11 de abril, por el que se aprueban las normas técnicas sobre condiciones de accesibilidad de los entornos urbanos, espacios públicos, edificaciones y sistemas de información y comunicación. (B.O.P.V. de 12-6-00).

\section{VALENCIA}

- Ley $1 / 1998$, de 5 de mayo, de Accesibilidad y Supresión de Barreras Arquitectónicas, Urbanísticas y de la Comunicación. (D.O.G.V. de 7-5-98). 


\section{CIUDAD AUTONOMA DE CEUTA}

- Ordenanza de 31 de marzo de 2003 para la accesibilidad y la eliminación de Barreras Arquitectónicas, Urbanísticas, del Transporte y de la Comunicación. (B.O.C.C. de 10-7-03).

Elaboración propia. Fuente: MINISTERIO DE SANIDAD Y POLITICA SOCIAL (2009): Servicio de Información sobre Discapacidad. Disponible en: sid.usal.es. Consultado en: 2009.

\section{REFERENCIAS BIBLIOGRÁFICAS}

CENTRO ESTATAL DE AUTONOMÍA, PERSONAS Y AYUDAS TÉCNICAS (CEAPAT) (1998): "Concepto Europeo de Accesibilidad". Boletín del Centro Estatal de Autonomía Personal y Ayudas Técnicas, $\mathrm{N}^{\circ}$ 22. Secretaría de Estado de Servicios Sociales, Familias y Discapacidad. Ministerio de Trabajo y Asuntos Sociales.

CENTRO ESTATAL DE AUTONOMÍA, PERSONAS Y AYUDAS TÉCNICAS (CEAPAT) (2001): "Resolución del Consejo Europeo sobre el Diseño Universal". Boletín del Centro Estatal de Autonomía Personal y Ayudas Técnicas, № 36, pp.2. Secretaría de Estado de Servicios Sociales, Familias y Discapacidad. Ministerio de Trabajo y Asuntos Sociales.

CENTRO ESTATAL DE AUTONOMÍA, PERSONAS Y AYUDAS TÉCNICAS (CEAPAT) (2001): "Resolución del Consejo Europeo sobre el Diseño Universal". Boletín del Centro Estatal de Autonomía Personal y Ayudas Técnicas, No 36, pp.2. Secretaría de Estado de Servicios Sociales, Familias y Discapacidad. Ministerio de Trabajo y Asuntos Sociales.

CENTRO ESTATAL DE AUTONOMÍA, PERSONAS Y AYUDAS TÉCNICAS (CEAPAT) (2003): "El Libro Blanco de la Accesibilidad". Boletín del Centro Estatal de Autonomía Personal y Ayudas Técnicas, № 45. pp. 2. Secretaría de Estado de Servicios Sociales, Familias y Discapacidad. Ministerio de Trabajo y Asuntos Sociales..

CENTRO ESTATAL DE AUTONOMÍA, PERSONAS Y AYUDAS TÉCNICAS (CEAPAT) (2003): "El Turismo en la Unión Europea". Boletín del Centro Estatal de Autonomía Personal y Ayudas Técnicas, $\mathrm{N}^{\circ}$ 45, pp.3-5. Secretaría de Estado de Servicios Sociales, Familias y Discapacidad. Ministerio de Trabajo y Asuntos Sociales.

COMISIÓN EUROPEA DG XXIII- UNIDAD DE TURISMO (1996): Por una Europa Accesible a Turistas con Discapacidades. Manual para la Industria del Turismo. CECA-CE-CEEA. Bruselas. Luxemburgo.

COMISIÓN EUROPEA (1991): “Por una Europa Accesible a Turistas con Discapacidades”. Disponible en: ec.europa.eu. Consultado en: 2010.

COMISIÓN EUROPEA (1996): "Comunicación sobre Igualdad de Oportunidades para personas con discapacidades". Disponible en: ec.europa.eu. Consultado en: 2010.

COMISIÓN EUROPEA (2003): "Feability Study-Comparable Statistics in the Area of Care of Dependent Adults in the European Union". Disponible en: http://europa.eu. Consultado en: 2010.

COMISIÓN EUROPEA (2003): "Criterios armonizados por una buena accesibilidad de los lugares e infraestructuras turísticas para las personas con discapacidad”. Dirección General de Empresas. Disponible en: http: \ Europa.eu.int. Consultado en: 2010.

COMITÉ ECONÓMICO Y SOCIAL EUROPEO (2003): Dictamen "Por un turismo accesible a todas las personas y socialmente sostenible". Disponible en: http://www.juntadeandalucia.es/ turismocomercioydeporte. Consultado en: 2010.

COMITÉ ESPAÑOL DE REPRESENTANTES DE PERSONAS CON DISCAPACIDAD (CERMI) (2003): Plan de Acción del CERMI de Turismo Accesible para todas las Personas. Madrid.

COMITÉ ESPAÑOL DE REPRESENTANTES DE PERSONAS CON DISCAPACIDAD (CERMI) (2005): Turismo Accesible. Hacia un Turismo para Todos. Madrid. 
CONSEJO DE EUROPA. COMITÉ DE MINISTROS (2001): "Resolución ResAP(2001) sobre Principios de Diseño Universal en los currícula de todas la actividades relacionadas con el entorno de la construcción". Adoptada por el Comité de Ministros de 15 de febrero de 2001, en la $742^{\text {a }}$ reunión de los Subsecretarios). Disponible en: sid.usal.es. Consultado en: 2009.

CONSEJO DE EUROPA. COMITÉ DE MINISTROS (2006): "Recomendación Rec(2006)5 del Comité de Ministros a los Estados miembros sobre el Plan de Acción del Consejo de Europa para la promoción de derechos y la plena participación de las personas con discapacidad en la sociedad: mejorar la calidad de vida de las personas con discapacidad en Europa 2006-2015”. Disponible en: sid.usal.es. Consultado en: 2008.

CONSTITUCIÓN ESPAÑOLA (1999): La Constitución Española de 1978. 2a ed., reimpresión. Civitas. Madrid.

DECLARACIÓN DE MADRID. “NO DISCRIMINACIÓN MÁS ACCIÓN POSITIVA ES IGUAL A INCLUSIÓN SOCIAL”. CONGRESO EUROPEO SOBRE LAS PERSONAS CON DISCAPACIDAD. 20-23 de marzo de 2002. Punto 1. Preámbulo. Madrid. Disponible en: www.discapnet.es/gdocumentos. Consultado en: Octubre de 2008.

DINARÉS QUERA, M. (2005): La promoción de la accesibilidad en otros países. Minusval. Disponible en: http://sid.usal.es/minusval.asp. Consultado en: 2008.

COMISIÓN EUROPEA (2007): La legislación en la Unión Europea. Consultado en: http: \\ Europa. eu.int. Consultado en: 2009.

FERNÁNDEZ ALLES, M.T. (2007): Turismo Accesible: Análisis de la Accesibilidad Hotelera en la Provincia de Cádiz. Servicio de Publicaciones de la Universidad de Cádiz. Cádiz.

HUESCA GONZÁLEZ, A.M. Y ORTEGA ALONSO, E. (2004): "Hábitos y actitudes hacia el turismo de las personas con discapacidad física. Informe de resultados 2004". Plataforma Representativa Estatal de Discapacitados Físicos (PREDIF). Madrid.

INSTITUTO DE MAYORES Y ASUNTOS SOCIALES (IMSERSO) (2002): "Normativa sobre Discapacidad en el año 2002". Minusval, Núm. 137. Servicio de Información sobre Discapacidad de la Secretaría de Estado de Servicios Sociales, Familia y Discapacidad. Ministerio de Trabajo y Asuntos Sociales. Noviembre-Diciembre. Madrid.

INSTITUTO DE MAYORES Y ASUNTOS SOCIALES (IMSERSO) (2003): Plan de Accesibilidad 2003-2010. ACCEPLAN. Libro Blanco. "Por un nuevo paradigma, el Diseño para Todos, hacia la plena igualdad de oportunidades". Ministerio de Trabajo y Asuntos Sociales. Instituto de Mayores y Asuntos Sociales. Ministerio de Trabajo y Asuntos Sociales. Madrid.

INSTITUTO DE MAYORES Y ASUNTOS SOCIALES (IMSERSO) (2003): I Plan Nacional de Accesibilidad 2004-2012. Secretaría General de Asuntos Sociales. Ministerio de Trabajo y Asuntos Sociales. Madrid.

INSTITUTO DE MAYORES Y ASUNTOS SOCIALES (IMSERSO) (2003): "Plan de Acción para las personas mayores 2003-2007”. Sesenta y Más. Número 219, Pp. 18-23. Septiembre. Ministerio de Trabajo y Asuntos Sociales. Secretaría de Estado de Servicios Sociales, Familias y Discapacidad. Madrid. Madrid.

INSTITUTO NACIONAL DE ESTADÍSTICA (2009): Encuesta de Discapacidad, Autonomía Personal y Situaciones de Dependencia (EDAD).

KOTLER, P.; ARMSTRONG, G; CÁMARA, D. Y CRUZ, I. (2004): Marketing. Pearson Prentice Hall. $12^{a}$ edición. Madrid.

KOTLER, P. Y ARMSTRONG, G. (2008): Fundamentos de Marketing. Prentice Hall. $8^{\text {a }}$ edición. Madrid.

KOTLER, P.; LANE, K; CÁMARA, D. Y MOLLÁ, A. (2006): Dirección de Marketing. Pearson Prentice Hall. $12^{\mathrm{a}}$ edición. Madrid.

LEY 13/1982, de 7 de abril, de Integración Social de los Minusválidos (LISMI). Disponible en: www.boe.es. Consultado en: 2008. 
LEY 51/2003, de 2 de diciembre, de Igualdad de Oportunidades, No Discriminación y Accesibilidad Universal de las Personas con Discapacidad. BOE núm. 289, de 3 de diciembre 2003. Disponible en: www.boe.es. Consultado en: 2008.

MARCOS PÉREZ, D. Y GONZÁLEZ VELASCO, D.J. (2003): Hacia un Turismo para Todos. Comité Español de Representantes de Personas con Discapacidad (CERMI). Instituto de Mayores y Servicios Sociales Secretaría General de Asuntos Sociales. Ministerio de Trabajo y Asuntos Sociales.

MINISTERIO DE SANIDAD Y POLITICA SOCIAL (2009): Servicio de Información sobre Discapacidad. Disponible en: sid.usal.es. Consultado en: 2009.

MINISTERIO DE TRABAJO Y ASUNTOS SOCIALES (2003): II Plan de Acción para las Personas con Discapacidad 2003-2007. Ministerio de Trabajo y Asuntos Sociales. Secretaría General de Asuntos Sociales. Madrid.

ORGANIZACIÓN MUNDIAL DEL TURISMO (O.M.T.) (1991): "Para un turismo accesible a los minusválidos en los años 90". Disponible en: http://www.unwto.org. Consultado en: 2010.

ORGANIZACIÓN MUNDIAL DE LA SALUD (O.M.S.) (2009): Sistema de Información Estadística de la OMS (WHOSIS). Disponible en: http://www.who.int. Consultado en: 2009.

ORGANIZACIÓN DE LAS NACIONES UNIDAS (1996): Normas Uniformes sobre la Igualdad de Oportunidades para las Personas con Discapacidad. Disponible en:http://www.un.org. Consultado en: 2008.

ORGANIZACIÓN DE LAS NACIONES UNIDAS (2006): La ONU y las Personas con Discapacidad. Disponible en:http://www.un.org/spanish/esa/social/disabled. Consultado en: 2008.

ORGANIZACIÓN DE LAS NACIONES UNIDAS PARA LA EDUCACIÓN, LA CIENCIA Y LA CULTURA (2006): Las Personas Discapacitadas. Disponible en: http://www.unesco.org. Consultado en: 2008.

PÉREZ BUENO, L.C. (2005): Mejora de la Accesibilidad Universal a los Entornos: propuesta del CERMI Estatal. Madrid. Comité Español de Representantes de Minusválidos. Madrid.

PLATAFORMA REPRESENTATIVA ESTATAL DE DISCAPACITADOS FISICOS (PREDIF) (2005): Hoteles Accesibles para Personas con Movilidad Reducida. Disponible en: www.predif. org. Consultado en: 2008. 